\title{
Two-Loop Superstrings in Hyperelliptic Language II: the Cosmological Constant and the Non-Renormalization Theorem
}

\author{
Zhu-Jun Zheng* \\ Institute of Mathematics, Henan University \\ Kaifeng 475001, P. R. China \\ and \\ Institute of Theoretical Physics, Chinese Academy of Sciences \\ P. O. Box 2735, Beijing 100080, P. R. China \\ Jun-Bao $\mathrm{Wu}$ \\ School of Physics, Peking University \\ Beijing 100871, P. R. China \\ Chuan-Jie Zhu ${ }^{\dagger}$ \\ Institute of Theoretical Physics, Chinese Academy of Sciences \\ P. O. Box 2735, Beijing 100080, P. R. China
}

November 10, 2018

*Supported in part by Math. Tianyuan Fund with grant Number 10226002 and the Natural Science Foundation of Educational Committee of Henan Province with grant Number 2000110010.

†Supported in part by fund from the National Natural Science Foundation of China with grant Number 90103004. 


\begin{abstract}
The vanishing of the cosmological constant and the non-renormalization theorem are verified at two loops by explicit computation using the hyperelliptic language and the newly obtained chiral measure of D'Hoker and Phong. A set of identities is found which is used in the verification of the non-renormalization theorem and leads to a great simplification of the calculation of the four-particle amplitude at two loops.
\end{abstract}

\title{
1 Introduction
}

Although we believe that superstring theory is finite in perturbation at any order 1, 2, 3, 4, a rigorous proof is still lacking despite great advances in the covariant formulation of superstring perturbation theory á la Polyakov. In particular, there is a non-renormalization theorem [4]. In spite of the efforts of many authors, it is very difficult to verify this theorem explicitly. Even in the case of the cosmological constant, i.e. the vacuum amplitude, this problem has not been completely solved. At two loops these problems were solved explicitly by using the hyperelliptic formalism in a series of papers [5, 6, 7, 8, 9. The explicit result was also used by Iengo [10, to prove the vanishing of perturbative correction to the $R^{4}$ term [11] at two loops, in agreement with the indirect argument of Green and Gutperle [12, Green, Gutperle and Vanhove [13, and Green and Sethi [14 that the $R^{4}$ term does not receive perturbative contributions beyond one loop. Recently, Stieberger and Taylor [15] also used the result of [8 to prove the vanishing of the heterotic two-loop $F^{4}$ term. For some closely related works we refer the reader to the reviews [16, 17]. In the general case, there is no satisfactory solution. For a review of these problem we refer the reader to [18, 19].

Recently two-loop superstring was studied by D'Hoker and Phong. In a series of papers [20, 21, 22, 23] (for a recent review see [19]), D'Hoker and Phong found an unambiguous and slice-independent two-loop superstring measure on moduli space for even spin structure from first principles.

Although their result is quite explicit, it is still a difficult problem to use it in actual computation. In [23], D'Hoker and Phong used their result to compute explicitly the chiral measure by choosing the split gauge and proved the vanishing of the cosmological constant and the non-renormalization theorem [24, 4]. They also computed the four-particle amplitude in another 
forthcoming paper [25]. Although the final results are exactly the expected, their computation is quite difficult to follow because of the use of theta functions. ${ }^{1}$ Also modular invariance is absurd in their computations because of the complicated dependence between the 2 insertion points (the insertion points are also spin structure dependent).

Although the vanishing of the cosmological constant and the non-renormalization theorem was proved explicitly in previous works [5, 6, 7, it would be interesting to study this problem again by using the newly obtained result of D'Hoker and Phong. The main purpose of this study is as a warm up exercise for the computation of the possibly non-vanishing four-particle amplitude. As we will see in this paper, some expressions are non-vanishing after summation over spin structures. Nevertheless the combination of the symmetry of the computed expression and the relevant kinematic factor gives a vanishing result. In a previous paper [27, we report the main results of our computation of two loop superstring theory by using hyperelliptic language. In this paper we will present the details for the proof of the vanishing of the cosmological constant and the non-renormalization theorem. The computation of the non-vanishing four-particle amplitude is given in another publication [28].

The organization of this paper is as follows. In the next section we will recall the relevant results of hyperelliptic representation of the genus 2 Riemann surface and set our notations for all the correlators. In section 3 we recall the results of D'Hoker and Phong for the chiral measure. In section 4 we computed explicitly all the relevant quantities in the chiral measure. Here we mainly concentrated on the spin structure dependent parts. In section 5 we established a set of identities and proved the vanishing of the cosmological constant. The identities will also be used in the next section in the verification of the non-renormalization theorem. Here modular invariance is maintained explicitly. In this section we also discuss the importance of taking the limit $\tilde{p}_{1} \rightarrow q_{1,2}$ and mention the (six) Riemann identities which are not fully modular invariant. In section 6 we proved the non-renormalization theorem. In particular we study carefully the most difficult part of the three-particle amplitude. Here the symmetry of the relevant kinematic factor is very important in the proof of the non-renormalization theorem. The (point-wise) vanishing of all the 1-, 2- and 3-particle amplitude leads a great simplification

\footnotetext{
${ }^{1}$ In [26], the two-loop 4-particle amplitude was also computed by using theta functions. Its relation with the previous explicit result 8 has not been clarified.
} 
of the computation of the 4-particle amplitude [28.

Here we note again that D'Hoker and Phong have proved that the cosmological constant and the 1-, 2- and 3-point functions are zero point-wise in moduli space [24. They have also computed the 4-particle amplitude [25]. The agreement of the results from these two different gauge choices and two different methods of computations is another proof of the validity of the new supersymmetric gauge fixing method at two loops.

\section{Genus 2 hyperelliptic Riemann surface}

First we remind that a genus-g Riemann surface, which is the appropriate world sheet for one and two loops, can be described in full generality by means of the hyperelliptic formalism. ${ }^{2}$ This is based on a representation of the surface as two sheet covering of the complex plane described by the equation:

$$
y^{2}(z)=\prod_{i=1}^{2 g+2}\left(z-a_{i}\right),
$$

The complex numbers $a_{i},(i=1, \cdots, 2 g+2)$ are the $2 g+2$ branch points, by going around them one passes from one sheet to the other. For twoloop $(g=2)$ three of them represent the moduli of the genus 2 Riemann surface over which the integration is performed, while the other three can be arbitrarily fixed. Another parametrization of the moduli space is given by the period matrix.

At genus 2, by choosing a canonical homology basis of cycles we have the following list of 10 even spin structures:

$$
\begin{array}{lll}
\delta_{1} \sim\left[\begin{array}{ll}
1 & 1 \\
1 & 1
\end{array}\right] \sim\left(a_{1} a_{2} a_{3} \mid a_{4} a_{5} a_{6}\right), & \delta_{2} \sim\left[\begin{array}{ll}
1 & 1 \\
0 & 0
\end{array}\right] \sim\left(a_{1} a_{2} a_{4} \mid a_{3} a_{5} a_{6}\right), \\
\delta_{3} \sim\left[\begin{array}{ll}
1 & 0 \\
0 & 0
\end{array}\right] \sim\left(a_{1} a_{2} a_{5} \mid a_{3} a_{4} a_{6}\right), & \delta_{4} \sim\left[\begin{array}{ll}
1 & 0 \\
0 & 1
\end{array}\right] \sim\left(a_{1} a_{2} a_{6} \mid a_{3} a_{4} a_{5}\right), \\
\delta_{5} \sim\left[\begin{array}{ll}
0 & 1 \\
0 & 0
\end{array}\right] \sim\left(a_{1} a_{3} a_{4} \mid a_{2} a_{5} a_{6}\right), & \delta_{6} \sim\left[\begin{array}{ll}
0 & 0 \\
0 & 0
\end{array}\right] \sim\left(a_{1} a_{3} a_{5} \mid a_{2} a_{4} a_{6}\right), \\
\delta_{7} \sim\left[\begin{array}{ll}
0 & 0 \\
0 & 1
\end{array}\right] \sim\left(a_{1} a_{3} a_{6} \mid a_{2} a_{4} a_{5}\right), & \delta_{8} \sim\left[\begin{array}{ll}
0 & 0 \\
1 & 1
\end{array}\right] \sim\left(a_{1} a_{4} a_{5} \mid a_{2} a_{3} a_{6}\right),
\end{array}
$$

\footnotetext{
${ }^{2}$ Some early works on two loops computation by using hyperelliptic representation are 29, 30, 31, 32, 33, 34, 35. which is by no means the complete list.
} 


$$
\delta_{9} \sim\left[\begin{array}{ll}
0 & 0 \\
1 & 0
\end{array}\right] \sim\left(a_{1} a_{4} a_{6} \mid a_{2} a_{3} a_{5}\right), \quad \delta_{10} \sim\left[\begin{array}{ll}
0 & 1 \\
1 & 0
\end{array}\right] \sim\left(a_{1} a_{5} a_{6} \mid a_{2} a_{3} a_{4}\right) .
$$

We will denote an even spin structure as $\left(A_{1} A_{2} A_{3} \mid B_{1} B_{2} B_{3}\right)$. By convention $A_{1}=a_{1}$. For each even spin structure we have a spin structure dependent factor from determinants which is given as follows [5]:

$$
Q_{\delta}=\prod_{i<j}\left(A_{i}-A_{j}\right)\left(B_{i}-B_{j}\right)
$$

This is a degree 6 homogeneous polynomials in $a_{i}$.

At two loops there are two odd supermoduli and this gives two insertions of supercurrent at two different points $x_{1}$ and $x_{2}$. Previously the chiral measure was derived in [36, 18, by a simple projection from the supermoduli space to the even moduli space. This projection does't preserve supersymmetry and there is a residual dependence on the two insertion points. This formalism was used in [5, 6, 7, 8. In these papers we found that it is quite convenient to choose these two insertion points as the two zeros of a holomorphic abelian differential which are moduli independent points on the Riemann surface. In hyperelliptic language these two points are the same points on the upper and lower sheet of the surface. We denote these two points as $x_{1}=x+$ (on the upper sheet) and $x_{2}=x-$ (on the lower sheet). We made these convenient choices again in 27] and will make the same choices in this paper and 28].

In the following we will give some formulas in hyperelliptic representation which will be used later. First all the relevant correlators are given by ${ }^{3}$

$$
\begin{aligned}
\left\langle\psi^{\mu}(z) \psi^{\nu}(w)\right\rangle & =-\delta^{\mu \nu} G_{1 / 2}[\delta](z, w)=-\delta^{\mu \nu} S_{\delta}(z, w), \\
\left\langle\partial_{z} X^{\mu}(z) \partial_{w} X^{\nu}(w)\right\rangle & =-\delta^{\mu \nu} \partial_{z} \partial_{w} \ln E(z, w), \\
\langle b(z) c(w)\rangle & =+G_{2}(z, w), \\
\langle\beta(z) \gamma(w)\rangle & =-G_{3 / 2}[\delta](z, w),
\end{aligned}
$$

where

$$
\begin{aligned}
& S_{\delta}(z, w)=\frac{1}{z-w} \frac{u(z)+u(w)}{2 \sqrt{u(z) u(w)}}, \\
& u(z)=\prod_{i=1}^{3}\left(\frac{z-A_{i}}{z-B_{i}}\right)^{1 / 2},
\end{aligned}
$$

\footnotetext{
${ }^{3}$ We follow closely the notation of 21$]$.
} 


$$
\begin{aligned}
& G_{2}(z, w)=-H(w, z)+\sum_{a=1}^{3} H\left(w, p_{1}\right) \varpi_{a}(z, z), \\
& H(w, z)=\frac{1}{2(w-z)}\left(1+\frac{y(w)}{y(z)}\right) \frac{y(w)}{y(z)}, \\
& G_{3 / 2}[\delta](z, w)=-P(w, z)+P\left(w, q_{1}\right) \psi_{1}^{*}(z)+P\left(w, q_{2}\right) \psi_{2}^{*}(z), \\
& P(w, z)=\frac{1}{\Omega(w)} S_{\delta}(w, z) \Omega(z),
\end{aligned}
$$

where $\Omega(z)$ is an abelian differential satisfying $\Omega\left(q_{1,2}\right) \neq 0$ and otherwise arbitrary. These correlators were adapted from [37. $\varpi_{a}(z, w)$ are defined in [20] and $\psi_{1,2}^{*}(z)$ are the two holomorphic $\frac{3}{2}$-differentials. When no confusion is possible, the dependence on the spin structure $[\delta]$ will not be exhibited.

In order take the limit of $x_{1,2} \rightarrow q_{1,2}$ we need the following expansions:

$$
\begin{aligned}
G_{3 / 2}\left(x_{2}, x_{1}\right) & =\frac{1}{x_{1}-q_{1}} \psi_{1}^{*}\left(x_{2}\right)-\psi_{1}^{*}\left(x_{2}\right) f_{3 / 2}^{(1)}\left(x_{2}\right)+O\left(x_{1}-q_{1}\right), \\
G_{3 / 2}\left(x_{1}, x_{2}\right) & =\frac{1}{x_{2}-q_{2}} \psi_{2}^{*}\left(x_{1}\right)-\psi_{2}^{*}\left(x_{1}\right) f_{3 / 2}^{(2)}\left(x_{1}\right)+O\left(x_{2}-q_{2}\right),
\end{aligned}
$$

for $x_{1,2} \rightarrow q_{1,2}$. By using the explicit expression of $G_{3 / 2}$ in (11) we have

$$
\begin{aligned}
& f_{3 / 2}^{(1)}\left(q_{2}\right)=-\frac{\partial_{q_{2}} S\left(q_{1}, q_{2}\right)}{S\left(q_{1}, q_{2}\right)}+\partial \psi_{2}^{*}\left(q_{2}\right) \\
& f_{3 / 2}^{(2)}\left(q_{1}\right)=\frac{\partial_{q_{1}} S\left(q_{2}, q_{1}\right)}{S\left(q_{1}, q_{2}\right)}+\partial \psi_{1}^{*}\left(q_{1}\right)=\left.f_{3 / 2}^{(1)}\left(q_{2}\right)\right|_{q_{1} \leftrightarrow q_{2}} .
\end{aligned}
$$

The quantity $\psi_{\alpha}^{*}(z)$ 's are holomorphic $\frac{3}{2}$-differentials and are constructed as follows:

$$
\psi_{\alpha}^{*}(z)=\left(z-q_{\alpha}\right) S\left(z, q_{\alpha}\right) \frac{y\left(q_{\alpha}\right)}{y(z)}, \quad \alpha=1,2 .
$$

For $z=q_{1,2}$ we have

$$
\begin{aligned}
& \psi_{\alpha}^{*}\left(q_{\beta}\right)=\delta_{\alpha, \beta}, \\
& \partial \psi_{1}^{*}\left(q_{2}\right)=-\partial \psi_{2}^{*}\left(q_{1}\right)=S\left(q_{1}, q_{2}\right)=\frac{i}{4} S_{1}(q), \\
& \partial \psi_{1}^{*}\left(q_{1}\right)=\partial \psi_{2}^{*}\left(q_{2}\right)=-\frac{1}{2} \Delta_{1}(q), \\
& \partial^{2} \psi_{1}^{*}\left(q_{1}\right)=\partial^{2} \psi_{2}^{*}\left(q_{2}\right)=\frac{1}{16} S_{1}^{2}(q)+\frac{1}{4} \Delta_{1}^{2}(q)+\frac{1}{2} \Delta_{2}(q),
\end{aligned}
$$


where

$$
\begin{aligned}
\Delta_{n}(x) & \equiv \sum_{i=1}^{6} \frac{1}{\left(x-a_{i}\right)^{n}} \\
S_{n}(x) & \equiv \sum_{i=1}^{3}\left[\frac{1}{\left(x-A_{i}\right)^{n}}-\frac{1}{\left(x-B_{i}\right)^{n}}\right]
\end{aligned}
$$

for $n=1,2$. This shows that $\partial \psi_{\alpha}^{*}\left(q_{\alpha+1}\right)$ and $\partial^{2} \psi_{\alpha}^{*}\left(q_{\alpha}\right)$ are spin structure dependent.

\section{The chiral measure: the result of D'Hoker and Phong}

The chiral measure obtained in [20, 21, 22, 23] after making the choice $x_{\alpha}=$ $q_{\alpha}(\alpha=1,2)$ is

$$
\begin{aligned}
\mathcal{A}[\delta] & =i \mathcal{Z}\left\{1+\mathcal{X}_{1}+\mathcal{X}_{2}+\mathcal{X}_{3}+\mathcal{X}_{4}+\mathcal{X}_{5}+\mathcal{X}_{6}\right\} \\
\mathcal{Z} & =\frac{\left\langle\prod_{a} b\left(p_{a}\right) \prod_{\alpha} \delta\left(\beta\left(q_{\alpha}\right)\right)\right\rangle}{\operatorname{det} \omega_{I} \omega_{J}\left(p_{a}\right)}
\end{aligned}
$$

and the $\mathcal{X}_{i}$ are given by:

$$
\begin{aligned}
& \mathcal{X}_{1}+\mathcal{X}_{6}=\frac{\zeta^{1} \zeta^{2}}{16 \pi^{2}}\left[-\left\langle\psi\left(q_{1}\right) \cdot \partial X\left(q_{1}\right) \psi\left(q_{2}\right) \cdot \partial X\left(q_{2}\right)\right\rangle\right. \\
&- \partial_{q_{1}} G_{2}\left(q_{1}, q_{2}\right) \partial \psi_{1}^{*}\left(q_{2}\right)+\partial_{q_{2}} G_{2}\left(q_{2}, q_{1}\right) \partial \psi_{2}^{*}\left(q_{1}\right) \\
&+\left.2 G_{2}\left(q_{1}, q_{2}\right) \partial \psi_{1}^{*}\left(q_{2}\right) f_{3 / 2}^{(1)}\left(q_{2}\right)-2 G_{2}\left(q_{2}, q_{1}\right) \partial \psi_{2}^{*}\left(q_{1}\right) f_{3 / 2}^{(2)}\left(q_{1}\right)\right] \\
& \mathcal{X}_{2}+\mathcal{X}_{3}= \frac{\zeta^{1} \zeta^{2}}{8 \pi^{2}} S_{\delta}\left(q_{1}, q_{2}\right) \\
& \quad \times \sum_{a=1}^{3} \tilde{\varpi}_{a}\left(q_{1}, q_{2}\right)\left[\left\langle T\left(\tilde{p}_{a}\right)\right\rangle+\tilde{B}_{2}\left(\tilde{p}_{a}\right)+\tilde{B}_{3 / 2}\left(\tilde{p}_{a}\right)\right] \\
& \mathcal{X}_{4}=\frac{\zeta^{1} \zeta^{2}}{8 \pi^{2}} S_{\delta}\left(q_{1}, q_{2}\right) \sum_{a=1}^{3}\left[\partial_{p_{a}} \partial_{q_{1}} \ln E\left(p_{a}, q_{1}\right) \varpi_{a}^{*}\left(q_{2}\right)\right. \\
&\left.\quad+\partial_{p_{a}} \partial_{q_{2}} \ln E\left(p_{a}, q_{2}\right) \varpi_{a}^{*}\left(q_{1}\right)\right]
\end{aligned}
$$




$$
\begin{aligned}
\mathcal{X}_{5}=\frac{\zeta^{1} \zeta^{2}}{16 \pi^{2}} & \sum_{a=1}^{3}\left[S_{\delta}\left(p_{a}, q_{1}\right) \partial_{p_{a}} S_{\delta}\left(p_{a}, q_{2}\right)\right. \\
& \left.-S_{\delta}\left(p_{a}, q_{2}\right) \partial_{p_{a}} S_{\delta}\left(p_{a}, q_{1}\right)\right] \varpi_{a}\left(q_{1}, q_{2}\right) .
\end{aligned}
$$

Furthermore, $\tilde{B}_{2}$ and $\tilde{B}_{3 / 2}$ are given by

$$
\begin{aligned}
\tilde{B}_{2}(w) & =-2 \sum_{a=1}^{3} \partial_{p_{a}} \partial_{w} \ln E\left(p_{a}, w\right) \varpi_{a}^{*}(w), \\
\tilde{B}_{3 / 2}(w) & =\sum_{\alpha=1}^{2}\left(G_{2}\left(w, q_{\alpha}\right) \partial_{q_{\alpha}} \psi_{\alpha}^{*}\left(q_{\alpha}\right)+\frac{3}{2} \partial_{q_{\alpha}} G_{2}\left(w, q_{\alpha}\right) \psi_{\alpha}^{*}\left(q_{\alpha}\right)\right) .
\end{aligned}
$$

In comparing with [22] we have written $\mathcal{X}_{2}, \mathcal{X}_{3}$ together and we didn't split $T(w)$ into different contributions. We also note that in eq. (26) the three arbitrary points $\tilde{p}_{a}(a=1,2,3)$ can be different from the three insertion points $p_{a}$ 's of the $b$ ghost field. The symbol $\tilde{\varpi}_{a}$ is obtained from $\varpi_{a}$ by changing $p_{a}$ 's to $\tilde{p}_{a}$ 's. In the following computation we will take the limit of $\tilde{p}_{1} \rightarrow q_{1}$. In this limit we have $\tilde{\varpi}_{2,3}\left(q_{1}, q_{2}\right)=0$ and $\tilde{\varpi}_{1}\left(q_{1}, q_{2}\right)=-1$. This choice greatly simplifies the formulas and also make the summation over spin structure doable (see below and [27, 28]).

\section{The chiral measure in hyperelliptic language}

The strategy we will follow is to isolate all the spin structure dependent parts first. As we will show in the following the spin structure dependent factors are just $S\left(q_{1}, q_{2}\right), \partial_{q_{2}} S\left(q_{1}, q_{2}\right)$ and the Szegö kernel if we also include the vertex operators. Before we do this we will first write the chiral measure in hyperelliptic language and take the limit of $\tilde{p}_{1} \rightarrow q_{1}$.

Let's start with $\mathcal{X}_{5}$. We have

$$
S\left(z, q_{1}\right) \partial_{z} S\left(z, q_{2}\right)-S\left(z, q_{2}\right) \partial_{z} S\left(z, q_{1}\right)=\frac{i}{4(z-q)^{2}} S_{1}(z) .
$$

So the spin structure dependent factor from $\mathcal{X}_{5}$ is effectively $S(z+, z-)$ as shown by the following formulas:

$$
\begin{aligned}
S\left(q_{1}, q_{2}\right) & =-S\left(q_{2}, q_{1}\right)=\frac{i}{4} S_{1}(q), \\
\partial_{q_{2}} S\left(q_{1}, q_{2}\right) & =-\partial_{q_{1}} S\left(q_{2}, q_{1}\right)=-\frac{i}{8} S_{2}(q) .
\end{aligned}
$$


For $\mathcal{X}_{4}$, the spin structure dependent factor is simply $S_{1}(q) \propto S\left(q_{1}, q_{2}\right)$ as $\ln E\left(p_{a}, q_{b}\right)$ and $\varpi_{a}^{*}\left(q_{b}\right)$ are spin structure independent (their explicit expressions are not needed in this paper and will be given in [28).

For $\mathcal{X}_{2}+\mathcal{X}_{3}$, we first compute the various contributions from the different fields. The total stress energy tensor is:

$$
\begin{aligned}
T(z)= & -\frac{1}{2}: \partial_{z} X(z) \cdot \partial_{z} X(z):+\frac{1}{2}: \psi(z) \cdot \partial_{z} \psi(z): \\
& -:\left(\partial b c+2 b \partial c+\frac{1}{2} \partial \beta \gamma+\frac{3}{2} \beta \partial \gamma\right)(z): \\
\equiv & T_{X}(z)+T_{\psi}(z)+T_{b c}(z)+T_{\beta \gamma}(z),
\end{aligned}
$$

in an obvious notations. The various contributions are

$$
\begin{aligned}
T_{X}(w) & =-10 T_{1}(w), \\
T_{\psi}(w) & =5 \tilde{g}_{1 / 2}(w)=\frac{5}{32}\left(S_{1}(w)\right)^{2}, \\
T_{b c}(w) & =\tilde{g}_{2}(w)-2 \partial_{w} f_{2}(w), \\
T_{\beta \gamma}(w) & =-\tilde{g}_{3 / 2}(w)+\frac{3}{2} \partial_{w} f_{3 / 2}(w),
\end{aligned}
$$

where

$$
\begin{aligned}
& f_{2}(w)=-\frac{3}{4} \Delta_{1}(w)+\sum_{a=1}^{3} H\left(w, p_{a}\right) \varpi_{a}(w, w), \\
& \tilde{g}_{2}(w)= \frac{5}{16} \Delta_{1}^{2}(w)+\frac{3}{8} \Delta_{2}(w) \\
&+\sum_{a=1}^{3} H\left(w, p_{a}\right) \varpi_{a}(w, w)\left(\frac{1}{w-p_{a+1}}+\frac{1}{w-p_{a+2}}-\Delta_{1}(w)\right), \\
& f_{3 / 2}(w)=\frac{\Omega^{\prime}(w)}{\Omega(w)}+\frac{\Omega\left(q_{1}\right)}{\Omega(w)} S\left(w, q_{1}\right) \psi_{1}^{*}(w)+\frac{\Omega\left(q_{2}\right)}{\Omega(w)} S\left(w, q_{2}\right) \psi_{2}^{*}(w), \\
& \tilde{g}_{3 / 2}(w)= \frac{1}{2} \frac{\Omega^{\prime \prime}(w)}{\Omega(w)}+\frac{1}{32}\left(S_{1}(w)\right)^{2} \\
&+\frac{\Omega\left(q_{1}\right)}{\Omega(w)} S\left(w, q_{1}\right) \partial \psi_{1}^{*}(w)+\frac{\Omega\left(q_{2}\right)}{\Omega(w)} S\left(w, q_{2}\right) \partial \psi_{2}^{*}(w) .
\end{aligned}
$$

As we said in the last section we will take the limit of $w \rightarrow q_{1}$. In this limit $T_{\beta \gamma}(w)$ is singular and we have the following expansion:

$$
T_{\beta \gamma}(w)=-\frac{3 / 2}{\left(w-q_{1}\right)^{2}}-\frac{\partial \psi_{1}^{*}\left(q_{1}\right)}{w-q_{1}}-\frac{1}{8} \Delta_{1}^{2}(q)-\frac{1}{32} S_{1}^{2}(q)+O\left(w-q_{1}\right) .
$$


The dependence on the abelian differential $\Omega(z)$ drops out. These singular terms are cancelled by similar singular terms in $\tilde{B}_{3 / 2}(w)$. By explicit computation we have:

$$
\begin{aligned}
\tilde{B}_{3 / 2}(w) & =\frac{3 / 2}{\left(w-q_{1}\right)^{2}}+\frac{\partial \psi_{1}^{*}\left(q_{1}\right)}{w-q_{1}}-\frac{1}{4} \Delta_{1}^{2}(q)+\frac{3}{4} \Delta_{2}(q) \\
- & \left(\frac{1}{p_{1}-q} \frac{\left(q-p_{2}\right)\left(q-p_{3}\right)}{\left(p_{1}-p_{2}\right)\left(p_{1}-p_{3}\right)} \Delta_{1}(q)+\ldots\right) \\
- & \frac{3}{2}\left(\frac{1}{\left(p_{1}-q\right)^{2}} \frac{\left(q-p_{2}\right)\left(q-p_{3}\right)}{\left(p_{1}-p_{2}\right)\left(p_{1}-p_{3}\right)}+\ldots\right)+O\left(w-q_{1}\right) .
\end{aligned}
$$

where $\ldots$ indicates two other terms obtained by cyclic permutating $\left(p_{1}, p_{2}, p_{3}\right)$. By using the above explicit result we see that the combined contributions of $T_{\beta \gamma}(w)$ and $\tilde{B}_{3 / 2}(w)$ are non-singular in the limit of $w \rightarrow q_{1}$. We can then take $\tilde{p}_{1} \rightarrow q_{1}$ in $\mathcal{X}_{2}+\mathcal{X}_{3}$. In this limit only $a=1$ contributes to $\mathcal{X}_{2}+\mathcal{X}_{3}$. This is because $\tilde{\varpi}_{2,3}\left(q_{1}, q_{2}\right)=0$ and $\tilde{\varpi}_{1}\left(q_{1}, q_{2}\right)=-1$. $T_{1}(w)$ and $T_{b c}(w)$ are regular in this limit and spin structure independent. In summary, the spin structure dependent factors from $\mathcal{X}_{2}+\mathcal{X}_{3}$ are the following two kinds (not including the vertex operators which will be consider later in section 6):

$$
S_{1}(q) \propto S\left(q_{1}, q_{2}\right), \quad \text { and } \quad\left(S_{1}(q)\right)^{3} .
$$

Here we note that if we don't take the limit of $w \rightarrow q_{1}$ (or $w \rightarrow q_{2}$ which has the same effect), the spin structure dependent factors from $\mathcal{X}_{2}+\mathcal{X}_{3}$ would be much more complicated. For example we will have a factor of the following kind:

$$
S_{1}(q)\left(S_{1}(w)\right)^{2} .
$$

The summation over spin structure with this factor will give a non-vanishing contribution as we will see later in eq. (171). We will discuss this point later in section 7 .

Finally we come to $\mathcal{X}_{1}+\mathcal{X}_{6}$. By using the explicit results given in eqs. (15)-(16), we have

$$
\begin{aligned}
\mathcal{X}_{1}+\mathcal{X}_{6}= & \left\langle\partial X\left(q_{1}\right) \cdot \partial X\left(q_{2}\right)\right\rangle S\left(q_{1}, q_{2}\right) \\
& -\left(\partial_{q_{1}} G_{2}\left(q_{1}, q_{2}\right)+\partial_{q_{2}} G_{2}\left(q_{2}, q_{1}\right)\right) S\left(q_{1}, q_{2}\right) \\
& +2\left(G_{2}\left(q_{1}, q_{2}\right)+G_{2}\left(q_{2}, q_{1}\right)\right) \\
& \times\left(\partial \psi_{1}^{*}\left(q_{1}\right) S\left(q_{1}, q_{2}\right)-\partial_{q_{2}} S\left(q_{1}, q_{2}\right)\right) .
\end{aligned}
$$


As $G_{2}\left(q_{1}, q_{2}\right)$ is spin structure independent, we see that all the spin structure dependent factors are the following two kinds:

$$
S\left(q_{1}, q_{2}\right)=\frac{i}{4} S_{1}(q)
$$

and

$$
\partial_{q_{2}} S\left(q_{1}, q_{2}\right)=\frac{i}{8} S_{2}(q)
$$

Here it is important that the factor $\partial \psi_{1}^{*}\left(q_{2}\right)$ cancels the factor $S\left(q_{1}, q_{2}\right)$ appearing in the denominator of $f_{3 / 2}^{(1)}\left(q_{2}\right)$.

From all the above results we see that all the spin structure dependent parts (for the cosmological constant) are as follows:

$$
c_{1} S_{1}(q)+c_{2} S_{2}(q)+c_{3} S_{1}^{3}(q)+\sum_{a=1}^{3} d_{a} S_{1}\left(p_{a}\right),
$$

where $c_{1,2,3}$ and $d_{a}$ 's are independent of spin structure. In computing the $n$-particle amplitude there are more spin structure factors coming from the correlators of $\psi$. We will include these terms when we discuss the nonrenormalization theorem.

\section{The vanishing of the cosmological constant}

The vanishing of the cosmological constant is proved by using the following identities:

$$
\begin{aligned}
& \sum_{\delta} \eta_{\delta} Q_{\delta} S_{n}(x)=0, \\
& \sum_{\delta} \eta_{\delta} Q_{\delta} S_{1}^{3}(x)=0,
\end{aligned}
$$

for $n=1,2$ and arbitrary $x$. Let us explain these identities in detail.

First we write down explicitly the simplest example:

$$
M(x, a)=\sum_{\delta} \eta_{\delta} \prod_{i<j}^{3}\left(A_{i}-A_{j}\right)\left(B_{i}-B_{j}\right) \sum_{k=1}^{3}\left[\frac{1}{x-A_{k}}-\frac{1}{x-B_{k}}\right] .
$$

By a Mobius transformation we have:

$$
\begin{aligned}
M(x, a) & =y^{4}(x) \sum_{\delta} \eta_{\delta} \prod_{i<j}^{3}\left(\tilde{A}_{i}-\tilde{A}_{j}\right)\left(\tilde{B}_{i}-\tilde{B}_{j}\right) \sum_{k=1}^{3}\left[\tilde{A}_{k}-\tilde{B}_{k}\right] \\
& \equiv y^{4}(x) M(\tilde{a}),
\end{aligned}
$$


where $\tilde{a}_{i}=\frac{1}{x-a_{i}}$.

As it was shown in [5], there is a unique set of phases $\eta_{\delta}$ for which $M(a)$ (and $M(x, a)$ ) is modular invariant in the following sense: for every interchanging $a_{i} \leftrightarrow a_{j}(i \neq j), M(a)$ got an overall "-" sign, i.e. $M(a)$ is antisymmetric for every interchange of the branch point $a_{i}$ 's. The phases are:

$$
\eta_{1}=-\eta_{2}=\eta_{3}=-\eta_{4}=\eta_{5}=-\eta_{6}=\eta_{7}=\eta_{8}=-\eta_{9}=\eta_{10}=1 .
$$

It is tedious to check explicitly that $M(a)$ is indeed antisymmetric for every interchange of the branch points by using the above set of phases. In doing so we see quite clearly that the factor $\sum_{k=1}^{3}\left[A_{k}-B_{k}\right]$ is also important because sometimes it also gives a "-" sign when we interchange $a_{1}$ with other branch points.

Here we remark that eq. (51) is still true if we neglect the factor $S_{1}(x)$. In fact these are exactly the Riemann identities for the $\theta$-constants by using the Thomae formula 38]:

$$
\Theta_{\delta}^{4}(0)= \pm \operatorname{det}^{2} K \prod_{i<j}^{3} A_{i j} B_{i j} .
$$

There are 6 set of phases which satisfies eq. (51). These correspond to the convention of setting $A_{1}$ to be any of the one fixed branch points, i.e. a choice of odd spin structures. As we can see from the above, a Riemann identity expression is not fully modular invariant and it is only invariant under the subgroup of modular transformations which leaves the fixed branch point invariant, i.e. any interchange of $a_{i} \leftrightarrow a_{j}$ but not with $A_{1}$. Even if the Riemann identities guarantees the vanishing of the cosmological constant if we blindly neglect the extra factors $S_{1}(x)$ and $S_{1}^{3}(x)$, they are not powerful enough to prove the non-renormalization theorem, not mentioning the explicit computation of the possibly non-vanishing 4-particle amplitude. (See more about this point at the end of this section.)

Now we proved that $M(a)$ is indeed modular invariant, it is trivial to prove that it is 0 . The trick is as follows (which is quite useful in what follows in the proof of non-renormalization theorem and the calculation of the four-particle amplitude). Because $M(a)$ is a homogeneous polynomial (of degree of 7) in $a_{i}$ and it is vanishing whenever $a_{i}=a_{j}$, it should be proportional to $P(a) \equiv \prod_{i<j}\left(a_{i}-a_{j}\right)$ which is a homogeneous polynomial of degree 15 in $a_{i}$. One see immediately that the power of $a_{i}$ can't be matched. 
So $M(a)$ must vanish. An explicit computation by computer also verifies this result. $^{4}$

The other identities in eq. (51) and eq. (52) can be proved similarly. We note that the power 3 in eq. (52) is important to make the expression modular invariant. In fact for all odd powers $n$, the following expression is modular invariant:

$$
M_{1, n}(x, a)=\sum_{\delta} \eta_{\delta} Q_{\delta} S_{1}^{n}(x)
$$

By power counting we have

$$
M_{1, n}(x, a)=0, \quad \text { for } \quad n=1,3,5,7 .
$$

$M_{1,9}(x, a)$ has the right power to be non-vanishing and we have

$$
M_{1,9}(x, a)=\frac{21 \times 2^{9} \times P(a)}{y^{6}(x)} .
$$

For $n=11$ the resulting summation is also quite simple and we have:

$$
M_{1,11}(x, a)=\frac{33 \times 2^{9} \times P(a)}{y^{6}(x)} \times\left(6 \Delta_{2}(x)-\Delta_{1}^{2}(x)\right) .
$$

For even $n$ we have the following results:

$$
\begin{aligned}
& M_{1,2}(x, a)=0 \\
& M_{1,4}(x, a)=\frac{32 P(a)\left(x-a_{1}\right)^{4}}{y^{4}(x) \prod_{i=2}^{6}\left(a_{1}-a_{i}\right)} .
\end{aligned}
$$

From the above results we see that although $M_{1,2 n}(x, a)$ is not modular invariant, it is invariant under a subgroup of the full modular transformation. This subgroup of modular transformations leaves $a_{1}$ fixed. This also explains why $M_{1,2}(x, a)$ is vanishing because it should proportional to a homogeneous polynomial $\tilde{P}(a)=\prod_{i<j=2}^{6}\left(a_{i}-a_{j}\right)$ which has degree 10 while $M_{1,2}(x, a)$ is only a homogeneous polynomial of degree 8 apart from the factor $y^{4}(x)$.

\footnotetext{
${ }^{4}$ Expanding $Q_{\delta}$ gives 36 different terms and multiplying with $\left(A_{1}+A_{2}+A_{3}-B_{1}-\right.$ $B_{2}-B_{3}$ ) gives 72 different terms. So we have 720 terms in the sum which must cancel each other.
} 


\section{The non-renormalization theorem}

For the non-renormalization theorem we need more identities. For graviton and the antisymmetric tensor the vertex operator is (left part only):

$$
V_{i}\left(k_{i}, \epsilon_{i}, z_{i}\right)=\left(\epsilon_{i} \cdot \partial X\left(z_{i}\right)+i k_{i} \cdot \psi\left(z_{i}\right) \epsilon_{i} \cdot \psi\left(z_{i}\right)\right) \mathrm{e}^{i k_{i} \cdot X\left(z_{i}, \bar{z}_{i}\right)} .
$$

By including the vertex operators we need to consider the following extra spin structure dependent terms:

$$
\begin{array}{ll}
\text { from } \mathcal{X}_{1}+\mathcal{X}_{6}: & \left\langle\psi\left(q_{1}\right) \psi\left(q_{2}\right) \prod_{i} k_{i} \cdot \psi\left(z_{i}\right) \epsilon_{i} \cdot \psi\left(z_{i}\right)\right\rangle \\
\text { from } \mathcal{X}_{2}+\mathcal{X}_{3}: & S_{1}(q)\left\langle: \psi\left(q_{1}\right) \cdot \partial \psi\left(q_{1}\right): \prod_{i} k_{i} \cdot \psi\left(z_{i}\right) \epsilon_{i} \cdot \psi\left(z_{i}\right)\right\rangle .
\end{array}
$$

The other terms are just the direct product of eq. (50) with the correlators from the vertex operators $\left\langle\prod_{i} k_{i} \cdot \psi\left(z_{i}\right) \epsilon \cdot \psi\left(z_{i}\right)\right\rangle$. Let's study these direct product terms (may be called as disconnected terms) first.

To prove the non-renormalization theorem we restrict our attention to 3 or less particle amplitude. For the 3-particle amplitude we have

$$
\left\langle\prod_{i=1}^{3} k_{i} \cdot \psi\left(z_{i}\right) \epsilon \cdot \psi\left(z_{i}\right)\right\rangle \propto S\left(z_{1}, z_{2}\right) S\left(z_{2}, z_{3}\right) S\left(z_{3}, z_{1}\right)+\text { (other terms). }
$$

By using the explicit expression of $S\left(z_{1}, z_{2}\right)$ we have

$$
\begin{array}{r}
S\left(z_{1}, z_{2}\right) S\left(z_{2}, z_{3}\right) S\left(z_{3}, z_{1}\right)=\frac{1}{8 z_{12} z_{23} z_{31}}\left\{2+\left[\frac{u\left(z_{1}\right)}{u\left(z_{2}\right)}+\frac{u\left(z_{2}\right)}{u\left(z_{1}\right)}\right]\right. \\
\left.+\left[\frac{u\left(z_{1}\right)}{u\left(z_{3}\right)}+\frac{u\left(z_{3}\right)}{u\left(z_{1}\right)}\right]+\left[\frac{u\left(z_{2}\right)}{u\left(z_{3}\right)}+\frac{u\left(z_{3}\right)}{u\left(z_{2}\right)}\right]\right\} .
\end{array}
$$

These factors combined with the other factors in eq. (501) give vanishing contribution to the $n$-particle amplitude by using the following "vanishing identities":

$$
\begin{aligned}
& \sum_{\delta} \eta_{\delta} Q_{\delta}\left\{\frac{u\left(z_{1}\right)}{u\left(z_{2}\right)}+\frac{u\left(z_{2}\right)}{u\left(z_{1}\right)}\right\} S_{n}(x)=0, \quad n=1,2 \\
& \sum_{\delta} \eta_{\delta} Q_{\delta}\left\{\frac{u\left(z_{1}\right)}{u\left(z_{2}\right)}-(-1)^{n} \frac{u\left(z_{2}\right)}{u\left(z_{1}\right)}\right\}\left(S_{1}(x)\right)^{n}=0, \quad n=2,3 .
\end{aligned}
$$


These identities can be proved by modular invariance and simple "power counting" which we have explained in detail in the last section.

Here we want to stress the importance of the limit $\tilde{p}_{1} \rightarrow q_{1,2}$. For arbitrary $\tilde{p}_{a}$, we would have a spin structure dependent factor $S_{1}(q)\left(S_{1}\left(\tilde{p}_{a}\right)\right)^{2}$ from $\mathcal{X}_{2,3}$ (specifically from $T_{\psi}$, and other terms from $T_{\beta \gamma}$ or $\tilde{B}_{3 / 2}\left(\tilde{p}_{a}\right)$ are more complicated as one can see from eqs. (41) and (42)). So we need to compute the following expression:

$$
\sum_{\delta} \eta_{\delta} Q_{\delta}\left\{\frac{u\left(z_{1}\right)}{u\left(z_{2}\right)}+\frac{u\left(z_{2}\right)}{u\left(z_{1}\right)}\right\} S_{1}(q)\left(S_{1}\left(\tilde{p}_{a}\right)\right)^{2} .
$$

Unfortunately the above expression is not identically 0 . We have:

$$
\begin{aligned}
\sum_{\delta} \eta_{\delta} Q_{\delta} S_{1}(x)\left\{\frac{u\left(z_{1}\right)}{u\left(z_{2}\right)}+\frac{u\left(z_{2}\right)}{u\left(z_{1}\right)}\right\}\left[\sum_{i=1}^{3}\left(A_{i}-B_{i}\right)\right]^{2} \\
=\frac{8 P(a)\left(x-z_{1}\right)\left(x-z_{2}\right)}{y^{2}(x) y\left(z_{1}\right) y\left(z_{2}\right)}\left(z_{1}-z_{2}\right)^{2}
\end{aligned}
$$

Our conjecture is that the combined result would still be 0 and independent of $\tilde{p}_{a}$ 's. Nevertheless the above limit of $\tilde{p}_{1} \rightarrow q_{1,2}$ greatly simplifies the algebra in the sense of making each term to be 0 identically. This limit also makes the computation of the four-amplitude doable (otherwise the algebra would be much more complicated). Now we turn our attention to the "disconnected" terms appearing in eqs. (64) and (65).

The terms in eq. (64) have already been discussed in 6]. Here we briefly review the argument. We have

$$
\left\langle\psi\left(q_{1}\right) \psi\left(q_{2}\right) \prod_{i} k_{i} \cdot \psi\left(z_{i}\right) \epsilon \cdot \psi\left(z_{i}\right)\right\rangle \propto S\left(q_{1}, z_{1}\right) S\left(z_{1}, z_{2}\right) S\left(z_{2}, z_{3}\right) S\left(z_{3}, q_{2}\right)+\cdots
$$

By using the explicit expression of $S(z, w)$ and note that $u\left(q_{2}\right)=-u\left(q_{1}\right)$ we have

$$
\begin{gathered}
S\left(q_{1}, z_{1}\right) S\left(z_{1}, z_{2}\right) S\left(z_{2}, z_{3}\right) S\left(z_{3}, q_{2}\right) \propto \sum_{i=1}^{3}\left[\frac{u\left(q_{1}\right)}{u\left(z_{i}\right)}-\frac{u\left(z_{i}\right)}{u\left(q_{1}\right)}\right] \\
+\sum_{i<j=1}^{3}\left[\frac{u\left(z_{i}\right)}{u\left(z_{j}\right)}-\frac{u\left(z_{j}\right)}{u\left(z_{i}\right)}\right]+\frac{u\left(q_{1}\right) u\left(z_{2}\right)}{u\left(z_{1}\right) u\left(z_{3}\right)}-\frac{u\left(z_{1}\right) u\left(z_{3}\right)}{u\left(q_{1}\right) u\left(z_{2}\right)} .
\end{gathered}
$$


These terms also give vanishing contributions as we can prove the following identities:

$$
\begin{aligned}
& \sum_{\delta} \eta_{\delta} Q_{\delta}\left\{\frac{u\left(z_{1}\right)}{u\left(z_{2}\right)}-\frac{u\left(z_{2}\right)}{u\left(z_{1}\right)}\right\}=0 \\
& \sum_{\delta} \eta_{\delta} Q_{\delta}\left\{\frac{u\left(z_{1}\right) u\left(z_{2}\right)}{u\left(z_{3}\right) u\left(z_{4}\right)}-\frac{u\left(z_{3}\right) u\left(z_{4}\right)}{u\left(z_{1}\right) u\left(z_{2}\right)}\right\}=0
\end{aligned}
$$

These identities were firstly proved in [6]. The proof is quite simple by using modular invariance. For example we have

$$
\begin{aligned}
& \sum_{\delta} \eta_{\delta} Q_{\delta}\left\{\frac{u\left(z_{1}\right)}{u\left(z_{2}\right)}-\frac{u\left(z_{2}\right)}{u\left(z_{1}\right)}\right\} \\
& =\frac{1}{y\left(z_{1}\right) y\left(z_{2}\right)} \sum_{\delta} \eta_{\delta} Q_{\delta}\left\{\prod_{i=1}^{3}\left(z_{1}-A_{i}\right)\left(z_{2}-B_{i}\right)-\prod_{i=1}^{3}\left(z_{1}-B_{i}\right)\left(z_{2}-A_{i}\right)\right\} \\
& \propto \frac{\left(z_{1}-z_{2}\right) P(a)}{y\left(z_{1}\right) y\left(z_{2}\right)}
\end{aligned}
$$

which must be vanishing as the degrees of the homogeneous polynomials (in $a_{i}$ and $z_{j}$ ) don't match. Here we have used again the modular invariance of the above expression. ${ }^{5}$

The last term we need to compute is the term in eq. (65). We have

$$
\begin{aligned}
&\langle: \psi\left.\left(q_{1}\right) \cdot \partial \psi\left(q_{1}\right): \prod_{i} k_{i} \cdot \psi\left(z_{i}\right) \epsilon \cdot \psi\left(z_{i}\right)\right\rangle_{c}=K(1,2,3) \\
& \quad \times\left(S\left(q_{1}, z_{1}, z_{2}, z_{3}\right)+S\left(q_{1}, z_{2}, z_{3}, z_{1}\right)+S\left(q_{1}, z_{3}, z_{1}, z_{2}\right)\right. \\
&\left.\quad-S\left(q_{1}, z_{1}, z_{3}, z_{2}\right)-S\left(q_{1}, z_{2}, z_{1}, z_{3}\right)-S\left(q_{1}, z_{3}, z_{2}, z_{1}\right)\right),
\end{aligned}
$$

\footnotetext{
${ }^{5}$ The minus sign in eq. (74) makes the expression invariant under the all the modular transformations. With a plus sign the expression is only invariant under a subgroup of the modular transformation. Nevertheless eq. (74) is still true with a plus sign. The explicit results are:

$$
\begin{aligned}
& \sum_{\delta} \eta_{\delta} Q_{\delta}\left\{\frac{u\left(z_{1}\right)}{u\left(z_{2}\right)}+\frac{u\left(z_{2}\right)}{u\left(z_{1}\right)}\right\}=0 \\
& \sum_{\delta} \eta_{\delta} Q_{\delta}\left\{\frac{u\left(z_{1}\right) u\left(z_{2}\right)}{u\left(z_{3}\right) u\left(z_{4}\right)}+\frac{u\left(z_{3}\right) u\left(z_{4}\right)}{u\left(z_{1}\right) u\left(z_{2}\right)}\right\}=\frac{2 P(a) z_{13} z_{14} z_{23} z_{24} \prod_{i=1}^{4}\left(a_{1}-z_{i}\right)}{\prod_{i=1}^{4} y\left(z_{i}\right) \prod_{i=2}^{6}\left(a_{1}-a_{i}\right)}
\end{aligned}
$$
}


where

$$
\begin{aligned}
K(1,2,3)= & k_{1} \cdot \epsilon_{3} k_{2} \cdot \epsilon_{1} k_{3} \cdot \epsilon_{2}-k_{1} \cdot \epsilon_{2} k_{2} \cdot \epsilon_{3} k_{3} \cdot \epsilon_{1} \\
& +k_{1} \cdot k_{2}\left(k_{3} \cdot \epsilon_{1} \epsilon_{2} \cdot \epsilon_{3}-k_{3} \cdot \epsilon_{2} \epsilon_{1} \cdot \epsilon_{3}\right) \\
& +k_{2} \cdot k_{3}\left(k_{1} \cdot \epsilon_{2} \epsilon_{3} \cdot \epsilon_{1}-k_{1} \cdot \epsilon_{3} \epsilon_{2} \cdot \epsilon_{1}\right) \\
& +k_{3} \cdot k_{1}\left(k_{2} \cdot \epsilon_{3} \epsilon_{1} \cdot \epsilon_{2}-k_{2} \cdot \epsilon_{1} \epsilon_{3} \cdot \epsilon_{2}\right), \\
S\left(x, z_{1}, z_{2}, z_{3}\right)= & S\left(x, z_{1}\right) S\left(z_{1}, z_{2}\right) S\left(z_{2}, z_{3}\right) \partial_{x} S\left(z_{3}, x\right) .
\end{aligned}
$$

We note that $K(1,2,3)$ is invariant under the cyclicly permutations of $(1,2,3)$. It is antisymmetric under the interchange $2 \leftrightarrow 3$. We have used these properties in eq. (79).

To compute explicitly these expressions we first note the following:

$$
\partial_{x} S(z, x)=\frac{1}{2(z-x)^{2}} \frac{u(z)+u(x)}{\sqrt{u(z) u(x)}}-\frac{S_{1}(x)}{8(z-x)} \frac{u(z)-u(x)}{\sqrt{u(z) u(x)}} .
$$

In order to do the summation over spin structure we need a "non-vanishing identity". This and other identities needed in the 4-particle amplitude computations are summarized as follows:

$$
\begin{aligned}
\sum_{\delta} \eta_{\delta} Q_{\delta} & \left\{\frac{u\left(z_{1}\right) u\left(z_{2}\right)}{u\left(z_{3}\right) u\left(z_{4}\right)}-(-1)^{n} \frac{u\left(z_{1}\right) u\left(z_{2}\right)}{u\left(z_{3}\right) u\left(z_{4}\right)}\right\}\left(S_{m}(x)\right)^{n} \\
& =\frac{2 P(a) \prod_{i=1}^{2} \prod_{j=3}^{4}\left(z_{i}-z_{j}\right) \prod_{i=1}^{4}\left(x-z_{i}\right)}{y^{2}(x) \prod_{i=1}^{4} y\left(z_{i}\right)} \times C_{n, m},
\end{aligned}
$$

where

$$
\begin{aligned}
C_{1,1}= & 1 \\
C_{2,1}= & -2\left(\tilde{z}_{1}+\tilde{z}_{2}-\tilde{z}_{3}-\tilde{z}_{4}\right) \\
C_{1,2}= & \Delta_{1}(x)-\sum_{k=1}^{4} \tilde{z}_{k}, \\
C_{3,1}= & 2 \Delta_{2}(x)-\Delta_{1}^{2}(x)+2 \Delta_{1}(x) \sum_{k=1}^{4} \tilde{z}_{k} \\
& +4 \sum_{k<l} \tilde{z}_{k} \tilde{z}_{l}-12\left(\tilde{z}_{1}+\tilde{z}_{2}\right)\left(\tilde{z}_{3}+\tilde{z}_{4}\right), \\
\tilde{z}_{k}= & \frac{1}{x-z_{k}}, \\
P(a)= & \prod_{i<j}\left(a_{i}-a_{j}\right) .
\end{aligned}
$$


$C_{1,1}$ and $C_{1,2}$ were derived in [8]. Although other values of $n, m$ also gives modular invariant expressions, the results are quite complex. ${ }^{6}$ Fortunately we only need to use the above listed results. The proof of these summation formulas will be given in 28].

By using these formulas we have:

$$
\sum_{\delta} \eta_{\delta} Q_{\delta} S\left(x, z_{1}, z_{2}, z_{3}\right) S_{1}(x)=-\frac{P(a)}{16 y^{2}(x)} \prod_{i=1}^{3} \frac{x-z_{i}}{y\left(z_{i}\right)} .
$$

We note that the above formula is invariant under the interchange $z_{i} \leftrightarrow z_{j}$.

By using this result and eq. (79), we have:

$$
\sum_{\delta} \eta_{\delta} Q_{\delta} S\left(q_{1}, q_{2}\right)\left\langle: \psi\left(q_{1}\right) \cdot \partial \psi\left(q_{1}\right): \prod_{i=1}^{3} k_{i} \cdot \psi\left(z_{i}\right) \epsilon_{i} \cdot \psi\left(z_{i}\right)\right\rangle_{\delta}=0 .
$$

This completes our verification of the non-renormalization theorem at two loops.

\section{Acknowledgments}

Chuan-Jie Zhu would like to thank Roberto Iengo for reading the paper and comments. He would also like to thank E. D'Hoker and D. Phong for discussions and Jian-Xin Lu and the hospitality at the Interdisciplinary Center for Theoretical Study, Physics, University of Science and Technology of China.

\section{References}

[1] M. B. Green and J. H. Schwarz, "Supersymmetric Dual String Theory III", Nucl. Phys. B198 (1982) 441.

[2] J. H. Schwarz, "Superstring Theory", Phys. Reports 89 (1982) 223322 .

[3] M. B. Green, "Lectures on Superstrings", in Unified String Theories, edited by M. Green and D. Gross (World Scientific, 1986).

\footnotetext{
${ }^{6}$ This is due to the non-vanishing of the summation over spin structures when we set $z_{1}=z_{3}$ or $z_{1}=z_{4}$, etc.
} 
[4] E. Martinec, "Nonrenormalization Theorems and Fermionic String Finiteness", Phys. Lett. 171B (1986) 189-194.

[5] E. Gava, R. Iengo and G. Sotkov, "Modular Invariance and the TwoLoop Vanishing of the Cosmological Constant", Phys. Lett. 207B (1988) 283-291.

[6] R. Iengo and C.-J. Zhu, "Notes on the Non-Renormalization Theorem in Superstring Theories", Phys. Lett. 212B (1988) 309-312.

[7] C.-J. Zhu, "Two-Loop Computations in Superstring Theories", Int. J. Mod. Phys. A4 (1989) 3877-3906.

[8] R. Iengo and C.-J. Zhu, "Two-Loop Computation of the Four-Particle Amplitude in Heterotic String Theory", Phys. Lett. 212B (1988) 313319.

[9] R. Iengo and C.-J. Zhu, "Explicit Modular Invariant Two-Loop Superstring Amplitude Relevant for $R^{4}$, J. High Energy Phys. 0006 (1999) 011, hep-th/9905050.

[10] R. Iengo, "Computing the $R^{4}$ Term at Two Superstring Loops", J. High Energy Phys. 0202 (2002) 035, hep-th/0202058.

[11] D. J. Gross and E. Witten, "Superstring Modification of Einstein's Equations", Nucl. Phys. B277 (1986) 1.

[12] M. B. Green and M. Gutperle, "Effects of D-instantons", Nucl. Phys. B498 (1997) 195-227, hep-th/9701093.

[13] M. B. Green, M. Gutperle and P. Vanhove, "One Loop in Eleven Dimension", Phys. Lett. 409B (1997) 277-184.

[14] M. B. Green and S. Sethi, "Supersymmetry Constraints on Type IIB Supergravity", Phys.Rev. D59 (1999) 046006, hep-th/9808061.

[15] S. Stieberger and T.R. Taylor, "Non-Abelian Born-Infeld action and type I - heterotic duality (I): Heterotic $F^{6}$ terms at two loops", Nucl.Phys. B647 (2002) 49-68, hep-th/0207026; "Non-Abelian BornInfeld Action and Type I - Heterotic Duality (II): Nonrenormalization Theorems", Nucl. Phys. B648 (2003) 3, hep-th/0209064. 
[16] M. B. Green, "Interconnections Between Type II Superstrings, M Theory and N=4 Supersymmetric Yang-Mills", hep-th/9903124.

[17] E. Kiritsis, "Duality and Instantons in String Theory",in Proceedings of the 1999 Spring Workshop on Superstrings and Related Matters, eds. B. Greene, J. Louis, K.S. Narain and S. Randjbar-Daemi (World Scientific, Singapore, 2000), hep-th/9906018.

[18] E. D'Hoker and D.H. Phong, "The Geometry of String Perturbation Theory", Rev. Mod. Phys. 60 (1988) 917-1065.

[19] E. D'Hoker and D.H. Phong, "Lectures on Two-Loop SuperstringsHangzhou, Beijing 2002", hep-th/0211111.

[20] E. D'Hoker and D.H. Phong, "Two-Loop Superstrings I, Main Formulas", Phys. Lett. B529 (2002) 241-255.

[21] E. D'Hoker and D.H. Phong, "Two-Loop Superstrings II, The chiral Measure on Moduli Space", Nucl. Phys. B636 (2002) 3-60.

[22] E. D'Hoker and D.H. Phong, "Two-Loop Superstrings III, Slice Independence and Absence of Ambiguities", Nucl. Phys. B636 (2002) 61-79.

[23] E. D'Hoker and D.H. Phong, "Two-Loop Superstrings IV, The Cosmological Constant and Modular Forms", Nucl. Phys. B639 (2002) $129-181$.

[24] E. D'Hoker and D.H. Phong, "Two-Loop Superstrings V, Scattering Amplitudes, the 1-, 2- and 3-point functions", in preparation.

[25] E. D'Hoker and D.H. Phong, "Two-Loop Superstrings VI, Scattering Amplitudes : the 4-point function", in preparation.

[26] O. Lechtenfeld and A. Parkes, "On Covariant Multiloop Superstring Amplitudes", Nucl. Phys. B332 (1990) 39-82.

[27] Zhu-Jun Zheng, Jun-Bao Wu and C.-J. Zhu, "Two-Loop Superstrings in Hyperelliptic Language I: the Main Results", hep-th/0212191. 
[28] Zhu-Jun Zheng, Jun-Bao Wu and C.-J. Zhu, "Two-Loop Superstrings in Hyperelliptic Language III: the Four-Particle Amplitude", hep-th/0212219.

[29] V. G. Knizhnik, "Explicit Expression for the Two-Loop Measure in the Heterotic String Theory", Phys. Lett. 196B (1987) 473-476.

[30] A. Morozov, "Two-Loop Statsum of Superstrings", Nucl. Phys. B303 (1988) 343-372; "On the Two-Loop Contribution to the Superstring Four-Point Function", Phys. Lett. 209B (1988) 473-476.

[31] A. Morozov and A. Pereomov, "Partition Functions in Superstring Theory. The Case of Genus Two", Phys. Lett. 197B (1987) 115-118; "A Note on Many-Loop Calculations for Superstrings in the NSR Formalism", Int. J. Mod. Phys. A4 (1989) 1773-1780.

[32] D. Lebdev and A. Morozov, "Statistical Sums of Strings on Hyperelliptic Riemann Surfaces", Nucl. Phys. B302 (1988) 163-188.

[33] O. Lechtenfeld and A. Parkes, "On the Vanishing of the Genus Two Superstring Vacuum Amplitude", Phys. Lett. 202B (1988) 75-80.

[34] M. A. Bershadsky, "The Superbranch Point and Two-Loop Correlation in Heterotic String Theory", Mod. Phys. Lett. A3 (1988) 91-108.

[35] G. Moore and A. Morozov, "Some Remarks on Two-Loop Superstring Calculations", Nucl. Phys. B306 (1988) 387-404.

[36] E. Verlinde and H. Verlinde, "Multiloop Calculations in Covariant Superstring Theory", Phys. Lett. 192B (1987) 95-102.

[37] M. Bonini and R. Iengo, "Correlation Functions and Zero Modes on Higher Genus Riemann Surface", Int. J. Mod. Phys. A3 (1988) 841860 .

[38] J. Fay, Theta Functions on Riemann Surfaces, Lecture Notes in Mathematics, vol. 352 (Springer-Verlag, Berlin, 1973). 